\title{
GNSS Multi-frequency Receiver Single-Satellite Measurement Validation Method
}

\author{
A. El-Mowafy \\ Dept. of Spatial Sciences, Curtin University, Australia \\ Tel:+61892663403 Fax: +61892662703 Email: a.el-mowafy@curtin,edu.au
}

\begin{abstract}
A method is presented for real-time validation of GNSS measurements of a single receiver, where data from each satellite are independently processed. A geometry-free observation model is used with a reparameterized form of the unknowns to overcome rank deficiency of the model. The ionosphere error and non-constant biases such as multipath are assumed changing relatively smoothly as a function of time. Data validation and detection of errors is based on statistical testing of the observation residuals using the Detection-Identification-Adaptation (DIA) approach. The method is applicable to any GNSS with any number of frequencies. The performance of validation method was evaluated using multiple-frequency data from three GNSS (GPS, GLONASS and Galileo) that span three days in a test site at Curtin University, Australia. Performance of the method in detection and identification of outliers in code observations and detection of cycle slips in phase data was examined. Results show that the success rate vary according to precision of observations and their number as well as size of the errors. The method capability is demonstrated when processing four IOV Galileo satellites in a single point positioning mode, and in another test by comparing its performance with Bernese software in detection of cycle slips in PPP processing using GPS data.
\end{abstract}

Keywords: Validation, Quality Control, GPS, Galileo, GLONASS, Multi-frequency GNSS.

\section{Introduction}

Successful GNSS software should include a pre-processing step for screening of data. During this pre-processing step the most severe irregularities in the data should be detected and if necessary repaired. Some techniques were presented for this purpose. For instance, the Receiver Autonomous Integrity Monitoring (RAIM) algorithms are generally based on checking consistency of solutions from different combinations of satellite data (Farrell and Van Graas 1992; Lee 2012). Other methods estimate cycle slips as additional unknowns in a least-squares or Kalman filtering processing (Banville and Langley 2010). Some methods used linear combinations of the observations or their time-difference to estimate cycle slips (Blewitt 1990, Dai 2012). The Detection-Identification-Adaptation (DIA) is another method for quality control of single-baseline GNSS observations, which has been discussed in Teunissen (1990), Teunissen (1998), and De Jong and Teunissen (2000). De Bakker el al. (2009a) used the DIA method to investigate quality control of single-receiver single-satellite geometry-free model with a focus on the analysis of the Minimal Detectable Bias (MDB), which is a measure for the size of the errors that can be detected with a certain power and probability of false alarm.

While most attention was given to validation of GPS observations, some studies consider multi-constellation GNSS. For instance, quality control of GPS with GLONASS was discussed in De Jong et al. (2001), and GPS with Galileo was considered in Ene et al. (2007); De Bakker el al. (2009b); and Neri et al. (2011). Most studies consider the case of dual-frequency

El-Mowafy, A. 2014. GNSS multi-frequency receiver single-satellite measurement validation method. GPS

Solutions. 18 (4): pp. 553-561.

Page 1 
observations due to the fact that signals availability was limited to only GPS and GLONASS (e.g Kim and Langley 2002). Some recent studies consider triple frequencies from GPS or Galileo (e.g. Guo et al. 2011). With the availability of new systems such as BeiDou, QZSS as well as Galileo, research is still needed in modelling and validation of quadruple or more frequency observations. In addition, as the number of visible satellites is increasing due to the presence of multi-constellation GNSS systems, there will be an increased likelihood that multiple outliers may occur in the collected observations at one epoch. Detection of multiple outliers in the data has been discussed in Kok (1984) and Teunissen (1990), and for RAIM in Blanch et. al. (2010).

In this contribution, a method is presented for validation of GNSS data using a single-receiver single-satellite approach and utilizing the DIA approach. The method is applicable for real-time or post-mission data processing. A re-parametrisation approach of the unknowns in a geometry-free model is presented and the used functional and dynamic modelling is discussed. The technique is applied for screening data of each satellite in an independent processing, i.e. one by one at each epoch and in a successive manner between epochs.

The paper is organized as follows. First, the multi-frequency single-receiver single-satellite method is discussed. Next, examples of its performance for processing data of a continuously operating reference station is presented. Data from three GNSS, namely GPS, GLONASS and Galileo, collected simultaneously for three consecutive days will be checked. Finally, the performance of the method is demonstrated and conclusions are given.

\section{Single-Receiver Single-satellite Geometry-free Modelling}

The carrier phase and pseudorange observation equations of a single receiver that tracks a single satellite on frequency $f_{j}$ (for $j=1$ to $n$ ) at time instant $t$ read (Teunissen and Kleusberg 1998; Leick 2004; Kaplan 2006):

$$
\begin{aligned}
& \phi_{j}(t)=\rho(t)+c\left(\delta t_{r}(t)-\delta t^{s}(t)\right)+T(t)-\mu_{j} I(t)+b_{\phi_{j}}(t)+\varepsilon_{\phi_{j}}(t) \\
& p_{j}(t)=\rho(t)+c\left(\delta t_{r}(t)-\delta t^{s}(t)\right)+T(t)+\mu_{j} I(t)+b_{p_{j}}(t)+\varepsilon_{p_{j}}(t)
\end{aligned}
$$

where $\phi_{j}(t)$ and $p_{j}(t)$ denote the observed carrier phase and pseudo ranges in distance units $(\mathrm{m})$, respectively, with corresponding zero-mean noise terms $\varepsilon_{\phi_{j}}(t)$ and $\varepsilon_{p_{j}}(t) . \rho(t)$ denotes the receiver-satellite range, $c$ is the speed of light, $\delta t_{r}(t)$ and $\delta t^{S}(t)$ are the receiver and satellite clock errors, and $T(t)$ is the tropospheric delay. The parameter $I(t)$ denotes the ionospheric delay for code observations and advance in phase observations expressed in units of distance with respect to the first frequency. For frequency $f_{j}$, the ionospheric coefficient $\mu_{j}=f_{1}^{2} / f_{j}^{2}$ is used to express its ionosphere in terms of $I(t)$. The parameters $b_{p_{j}}(t)$ and $b_{\phi_{j}}(t)$ are the code and phase biases (including the phase ambiguity) at epoch $(t)$, respectively. A geometry-free approach is used where positioning is of no interest at this stage. Thus, the satellite orbit error is not present in the model as knowledge of the accurate $3 \mathrm{D}$ satellite position is not needed. The ionospheric delay $I(t)$ can be decomposed into two components; its initial value $I\left(t_{o}\right)$ and the difference from this value, which is denoted as $(\delta I)$, such that:

$I(t)=I\left(t_{o}\right)+\delta I(t)$

El-Mowafy, A. 2014. GNSS multi-frequency receiver single-satellite measurement validation method. GPS 
where $t_{o}$ refers to the initial epoch of data processing.

The model given in Eq. (1) shows that the problem at hand is under-determined. The rank-defect is caused by the fact that the information content of the observables is such that only time-differences of the parameters can be determined. The rank deficiency in the model solution is reduced by re-parameterisation of the unknowns in the observation equations as follows (El-Mowafy et al. 2010):

$\rho^{*}(t)=\rho(t)+c\left(\delta t_{r}(t)-\delta t^{s}(t)\right)+T(t)$

$\rho^{* *}(t)=\rho^{*}(t)-\rho^{*}\left(t_{o}\right)$

$b_{\phi_{j=1 \text { to } n}^{*}}^{*}(t)=b_{\phi_{j=1 \text { to } n}}(t)+\left[\rho^{*}\left(t_{o}\right)-\mu_{j=1 \text { to } n} I\left(t_{o}\right)\right]$

$b_{p_{j=1 \text { ton } n}^{*}}^{*}(t)=b_{p_{j=1 \text { ton }}}(t)+\left[\rho^{*}\left(t_{o}\right)+\mu_{j=1 \text { to } n} I\left(t_{o}\right)\right]$

The observation equations in terms of the re-parameterized vector of unknowns $\left(\rho^{* *}(t)\right.$, $\left.\delta I(t), b_{\phi_{j=1 \text { to } ~}^{*}}^{*}(t), b_{p_{j=1 \text { to } ~}^{*}}^{*}(t)\right)^{T}$ at time $(t)$ then read:

$\phi_{j=1 \text { to } n}(t)=\rho^{* *}(t)-\mu_{j=1 \text { to } n} \delta I(t)+b_{\phi_{j=1 \text { ton }}^{*}}(t)+\varepsilon_{\phi_{j=1 \text { to } n}}(t)$

$p_{j=1 \text { to } n}(t)=\rho^{* *}(t)+\mu_{j=1 \text { to } n} \delta I(t)+b_{p_{j=1 \text { to } n}^{*}}^{*}(t)+\varepsilon_{p_{j=1 \text { to } n}}(t)$

During initialization when processing, the first two terms on the right-hand side of the equation equal zeros at the first epoch $\left(t_{o}\right)$, leading to $b_{\phi_{j}}^{*}\left(t_{o}\right)$ and $b_{p_{j}}^{*}\left(t_{o}\right)$ equal $\phi_{j}\left(t_{o}\right)$ and $p_{j}\left(t_{o}\right)$, for frequency $j$, respectively. The rank deficiency is completely removed by predicting the unknowns in Eq. (7), as will be discussed in the next section, and treating the predicted unknowns as pseudo observations. At time $t$ for the unknown vector $\left[\rho^{* *}(t), \delta I(t)\right.$, $\left.b_{\phi_{j=1 \text { to } n}}^{*}(t), b_{p_{j=1 \text { to } n}}^{*}(t)\right]^{T}$ denoted in a general form as $x_{t}$ we have:

$\tilde{x}_{t}=\Phi_{t / t-1} \hat{x}_{t-1}$

where $\Phi_{t / t-1}$ is the transition matrix, $\hat{x}_{t-1}$ and $\tilde{x}_{t}$ are the estimated and predicted vectors of unknowns at times $t-1$ and $t$. The reparametrized unknown range $\left(\rho^{* *}\right)$ is in general hard to predict unless the satellite and receiver motion and dynamics are taken into consideration. One way to avoid the complexity of this scenario is to model $\left(\rho^{* *}\right)$ using a random walk process. However, this process requires good stochastic information and one has also to consider the fact that a random walk process noise increases with time. Alternatively, the reparametrized unknown range $\left(\rho^{* *}\right)$ can be considered unlinked in time and thus excluded from the prediction process, such that the predicted vector of unknowns reads:

$\hat{g}_{t}=M \tilde{x}_{t}$

where $M$ is a diagonal identity matrix except that the first element equals zero, such that $\mathrm{M}=$ $\operatorname{diag}\left[0,1, \mathrm{I}_{2 n \times 2 n}\right]$ (Teunissen, personal communication) and $\hat{g}_{t}=\left[\delta I(t), b_{\phi_{j=1 \text { ton }}}^{*}(t)\right.$, $\left.b_{\phi_{j=1 \text { ton }}}^{*}(t)\right]^{T}$. Processing can be performed using Kalman filtering, however, this needs some manpulation of its formulation to account for the use of $\hat{g}_{t}$ instead of $\tilde{x}_{t}$. Equivalently, one can use a parametric least-squares adjustment, processing epoch by epoch, and augmenting the observation model by using the predicted unknowns as pseudo observations, such that: 
$\left[\begin{array}{l}y_{t} \\ \hat{g}_{t}\end{array}\right]=\left[\begin{array}{l}A_{t}^{\prime} \\ M\end{array}\right] x_{t}+\varepsilon_{t}$

with $A_{t}^{\prime}=\left[\begin{array}{llll}u & -\mu_{j=1 \text { to } n} & \mathrm{I} & 0 \\ u & +\mu_{j=1 \text { to } n} & 0 & \mathrm{I}\end{array}\right]$, where $y_{t}$ is the vector of observations, which comprises code and phase observations, $u$ is a column vector of ones with a size $n, \mu_{j=1 \text { to } n}$ is a column vector of size $n$, I is the identity matrix of size $n$, and $\varepsilon_{t}$ denotes a zero-mean vector of observation noise. The estimated values of the unknowns $\left(\hat{x}_{t}\right)$ can then be determined as follows:

$\hat{x}_{t}=\left[A_{t}^{\prime T} Q_{y_{t}}^{-1} A_{t}^{\prime}+M_{t}^{T} Q_{\hat{g}_{t / t-1}}^{-1} M_{t}\right]^{-1}\left[A_{t}^{\prime T} Q_{y_{t}}^{-1} y_{t}+M_{t}^{T} Q_{\hat{g}_{t / t-1}}^{-1} \hat{g}_{t / t-1}\right]$

\section{Dynamic and Stochastic Modelling}

In Eq. (7), the ionospheric delay $\delta I$ and the bias components $b_{\phi_{j}}^{*}$ and $b_{p_{j}}^{*}$ are assumed changing relatively smoothly with time for a short period (El-Mowafy 2009), which can be assumed between 15 and 30 minutes, depending on site and observing conditions. The temporal correlations of the three biases, denoted in a general term as $\beta$, are assumed exponentially decaying with time by using a first-order autoregressive stochastic process, such that:

$\beta=e^{-|\Delta t| / \tau}$

where $\Delta t$ is the time interval between the epochs $(t-1)$ and $(t)$ and $\tau$ is the correlation time length. The dynamic models of $\delta I, b_{\phi_{j}}^{*}$ and $b_{p_{j}}^{*}$ for a frequency $j$ at $t$ then read:

$\delta I(t)=\beta_{\delta I} \delta I(t-1)+d_{\delta I}(t)$

$b_{\phi_{j}}^{*}(t)=\beta_{b_{\phi_{j}}^{*}} b_{\phi_{j}}^{*}(t-1)+d_{b_{\phi_{j}}^{*}}(t)$

$b_{p_{j}}^{*}(t)=\beta_{b_{p_{j}}^{*}} b_{p_{j}}^{*}(t-1)+d_{b_{p_{j}}^{*}}(t)$

where $\beta_{\delta I}, \beta_{b_{\phi_{j}}^{*}}$ and $\beta_{b_{p_{j}}^{*}}$ are the temporal correlations for $\delta I(t), b_{\phi_{j}}^{*}(t)$ and $b_{p_{j}}^{*}(t)$. $d_{\delta I}(t), d_{b_{\phi_{j}}^{*}}(t)$ and $d_{b_{p_{j}}^{*}}(t)$ are their process noises, which are assumed Gaussian white noises. The transition matrix in Eq. (8) then reads:

$\Phi_{t / t-1}=\operatorname{diag}\left[\beta_{\delta I}, \beta_{b_{\phi_{j=1 . . n}}^{*}}, \beta_{b_{p_{j=1 . . n}^{*}}^{*}}\right]$

Where $\beta_{b_{\phi_{j=1 . n}}^{*}}$ and $\beta_{b_{p_{j=1 . . n}}^{*}}$ are diagonal matrices including the values of $\beta_{b_{\phi_{j}}^{*}}$ and $\beta_{b_{p_{j}}^{*}}$ for each frequency, where $j=1$ to $n$. The variance of each process noise is $\left[\frac{\vartheta}{2 / \tau}\left(1-\beta^{2}\right)\right]($ Gelb et al. 1974), where $\vartheta$ denotes its spectral density. No auto-correlation nor cross-correlation among code and phase measurements are assumed in the used stochastic model. 


\section{Validation of the Observations Using the Single-Receiver Single-Satellite Model}

For detection of outliers in the observations, one may consider examining the model using observations only from the current epoch $(t)$. This is referred to as Local Testing. In local validation of GNSS observations, one may wish to test $q$ number of possible errors in the observations, where $q<d f$, where $d f$ is the degrees of freedom of the model. For $u$ number of unknowns, and excluding the unknown $\rho^{* *}$ during the prediction process, the number of predicted unknowns is $u-1$. Thus, the degrees of freedom for $n$ number of observed frequencies are $[2 n+(u-1)]-u]=2 n-1$. The best estimator of the error vector $\left(\widehat{\nabla}_{t}\right)$ can be determined from (Teunissen 2006):

$\widehat{\nabla}_{t}=\left(C_{t}^{T} Q_{y_{t}}^{-1} Q_{\hat{e}_{t}} Q_{y_{t}}^{-1} C_{t}\right)^{-1} C_{t}^{T} Q_{y_{t}}^{-1} \hat{e}_{t}$

and its covariance matrix is:

$Q_{\widehat{\nabla}_{t}}=\left(C_{t}^{T} Q_{y_{t}}^{-1} Q_{\hat{e}_{t}} Q_{y_{t}}^{-1} C_{t}\right)^{-1}$

where $\hat{e}_{t}$ and $Q_{\hat{e}_{t}}$ are the computed observation residuals and their covariance matrix from the least squares solution, where:

$\hat{e}_{t}=Y_{t}-A_{t} \hat{x}_{t}=\left[\begin{array}{l}y_{t} \\ \hat{g}_{t}\end{array}\right]-\left[\begin{array}{c}A_{t}^{\prime} \\ M\end{array}\right] \hat{x}_{t}$

and $Q_{\hat{e}_{t}}=Q_{Y_{t}}-\left[A_{t}\left(A_{t}^{T} Q_{Y_{t}}^{-1} A_{t}\right)^{-1} A_{t}^{T}\right]$, with $A_{t}=\left[\begin{array}{c}A_{t}^{\prime} \\ M\end{array}\right]$, and $Q_{Y_{t}}=\left[\begin{array}{cc}Q_{y_{t}} & 0 \\ 0 & Q_{\hat{g}_{t}}\end{array}\right]$ is the covariance matrix of the observations and predicted unknowns. $C_{t}$ is the matrix describing which observations are examined, such that each column of $C_{t}$ describes one possible error. The elements of each column are set to zeros except the element corresponding to the examined observation, which equals 1 .

Possible detection of the presence of model errors in local testing can be performed by examining the Local Over-all Model (LOM) statistic $T_{L O M}$, which can be formulated as (Teunissen 2006):

$T_{L O M}=\widehat{\nabla}_{t}^{T} Q_{\widehat{\nabla}_{t}}^{-1} \widehat{\nabla}_{t}$

and measurement or model errors are suspected when:

$T_{L O M} \geq \chi_{\alpha}^{2}(d f, 0)$

where $\chi_{\alpha}^{2}$ is the Chi-squared value for a significance level $\alpha$.

Once the presence of model errors is detected, one needs to identify the erroneous measurement(s) that cause such model errors. The matrix $C_{t}$ is set to test all possibilities of the presence of errors in the observations. For local testing, two cases are of particular interest:

1- The case of a single outlier in one code or phase observation, i.e. $q=1$. In this case, the $C_{t}$ matrix reduces to a column vector $c_{t}, \widehat{\nabla}_{t}$ becomes a scalar, and the test statistic can be computed as follows (Baarda 1968):

El-Mowafy, A. 2014. GNSS multi-frequency receiver single-satellite measurement validation method. GPS 
$w_{t}=\frac{\widehat{\nabla}_{t}}{\sigma_{\widehat{\nabla}_{t}}}$

where $\sigma_{\widehat{\nabla}_{t}}$ is the standard deviation of $\widehat{\nabla}_{t}$. The null hypothesis, denoted as $H_{o}$, is set to present the case that no outliers are present in the data. $H_{o}$ is rejected if

$\left|w_{t}\right| \geq N_{\frac{\alpha}{2}}(0,1)$

2- The case of multiple outliers, or complete loss of lock either in phase or in code observations, i.e. when $1<q \leq d f$. In this case, $C_{t}$ is a matrix with a number of columns equals $q$.

For the cases mentioned above, where different alternative hypotheses are examined, we have mixed size cases (i.e. $q=1$ for a single outlier and $q>1$ for the case of multiple outliers). Therefore, a unified criterion needs to be set to compare the statistical testing outcomes of different alternative hypotheses in order to identify possible observations that may contain the errors. This can be performed by comparing the P-values under the $\chi^{2}$ distribution for different alternative hypotheses (where P-value is the probability of obtaining a test statistic at least as extreme as the observed one). All alternative hypotheses are ranked in a descending order according to their P-values, where the alternative hypothesis that has the smallest P-value is considered as the most likely alternative hypothesis. For the case of $q=1$, the P-value of $w_{t}^{2}$ is computed as it has a $\chi^{2}$ distribution.

In identification testing, one has to consider the correlations among observation errors as this may introduce type III errors (identification of the wrong observations that do not include the outliers and missing the faulty ones if they have significant correlation). For observations $i$ and $j$, and ignoring the time index, the correlation coefficient between their corresponding errors denoted as $\xi_{\widehat{\nabla}_{i}, \widehat{\nabla}_{j}}$ reads:

$\xi_{\widehat{\nabla}_{i}, \widehat{\nabla}_{j}}=\frac{c_{i}^{T} Q_{Y}^{-1} Q_{\widehat{e}} Q_{Y}^{-1} c_{j}}{\sqrt{c_{i}^{T} Q_{Y}^{-1} Q_{\widehat{e}} Q_{Y}^{-1} c_{i}} \sqrt{c_{j}^{T} Q_{Y}^{-1} Q_{\widehat{e}} Q_{Y}^{-1} c_{j}}}$

where $c_{i}$ and $c_{j}$ are zero column vectors except for the elements corresponding to the observations $i$ and $j$ which equal 1 . If single frequency observations are used in the given model, the correlation between phase and code observation errors is almost -1 , and identification is not possible. For multi-frequency data, the correlation between phase errors is nearly -1 whereas that between code observation errors is almost zero. This means that phase errors will be hard to identify due to their high correlation whereas error identification will be possible for code errors since there is no correlation between them. In this study, we will restrict attention to detection and identification of outliers in code observations in the local testing case and detection of cycle slips in phase observations. To detect cycle slips, more than one epoch of data have to be examined. This is referred to as Global testing, where the Global Over-all Model (LOM) statistic $T_{G O M}$ reads:

$T_{G O M}=\sum_{i=t-\delta t}^{t} \widehat{\nabla}_{i}^{T} Q_{\widehat{\nabla}_{i}}^{-1} \widehat{\nabla}_{i}$

where $\delta t$ denotes to the time interval considered in computation of $T_{G O M}$ (e.g. two or three

El-Mowafy, A. 2014. GNSS multi-frequency receiver single-satellite measurement validation method. GPS

Solutions. 18 (4): pp. 553-561.

Page 6 
epochs). The $T_{G O M}$ statistic has also a Chi-squared distribution under $H_{o}$ and cycle slips are suspected in phase observations if this statistic exceeds a critical value of Chi-squared using the chosen significance level and degrees of freedom that are computed from the accumulated observations. Once a cycle slip is detected, the validation procedure is re-initialized.

\section{Testing the Single-Receiver Single-satellite Validation of GNSS Measurements}

The previous sections summarize the single-receiver single-satellite approach for vaildation of GNSS data. This approach has the following advantages:

- No satellite positions need to be known beforehand and thus no complete navigation messages need to be read and used. In this case, observation weighting can be performed using, for instance, the signal-to-noise ratio.

- Measurements from systems with a limited number of operational satellites, such as Galileo and QZSS, can be screened without the need for having a complete positioning solution.

- There is no need for the determination of inter-system biases when using data from different constellations.

In addition, the approach has the following capabilities:

- It can detect multi-faults at any one epoch for each satellite.

- Fault detection can be performed for a single or multi-frequency observations.

- Due to the method flexibility, it can be applied to any receiver type and make, and under static or kinematic modes.

In this section, practical testing of the proposed method is carried out. Description of the test data and assumptions are first given. Next, evaluation of the performance of the method is discussed, first for detection and identification of outliers in code observations in a local testing, and next for detection of cycle slips in phase observations.

\section{Test Description}

The single-receiver single-satellite method is tested using data that span three days as a representative sample. The observations were collected in a static mode at a continuously operating reference station (CORS) at Curtin University, Western Australia, during the period $15 / 3 / 2012$ to $17 / 3 / 2012$ with 30 seconds sampling interval. Observations from GPS, GLONASS and Galileo were collected using a geodetic-grade multi-frequency multi-GNSS antenna (TRM59800.00) and receiver (Septentrio POLARX4). Tracked signals in the test included L1, L2 and L5 code and phase observations for GPS, L1 and L2 for GLONASS, and E1, E5a and E5b for Galileo. Over each day, 32 GPS satellites, 24 GLONASS satellites, and 4 Galileo satellites (including GIOVE A and B, and IOV PRN 11 and 12) were observed.

An indication on the correctness of the model and assumed observation stochastic information used can be obtained by examining whether the estimated w-test statistic of the observed signals has a standard normal distribution as an incorrect model or inappropriate stochastic assumptions would lead to a wrong distribution. This can be performed by inspection of the probability plots of the w-test statistic. In this plot, the data are ordered and plotted against the correspondeing percentage points from a standard normal distribution in such a way that the points should form an approximate straight line. Departures from this straight line indicate departures from normality. An example of tested normal probability plots is given in Figure 1 for $p_{1}$ code observations of GLONASS satellite PRN 18 collected on

El-Mowafy, A. 2014. GNSS multi-frequency receiver single-satellite measurement validation method. GPS

Solutions. 18 (4): pp. 553-561.

Page 7 
$15 / 3 / 2012$. In our tests, the observations were weighted using an elevation-angle dependent model in the form $\left[1+10 \times e^{\left(-E^{o} / 10^{\circ}\right)}\right.$ (Euler and Goad 1991, Teunissen and de Bakker 2012), where $E^{o}$ is the observed elevation angle. The standard deviations used for the undifferenced observations were selected according to the observation type (phase/code), frequency and constellation and to satisfy the condition that the w-test statistic of each observed signal has a standard normal distribution. These standard deviations along zenith direction agreed in general with the values given in the litrature. For $\delta I, b_{\phi_{j}}^{*}$ and $b_{p_{j}}^{*}$, the used spectral densities were $3 \mathrm{~mm}^{2} / \mathrm{s}, 2 \mathrm{~mm}^{2} / \mathrm{s}$ and $50 \mathrm{~mm}^{2} / \mathrm{s}$, with correlation times $1500 \mathrm{~s}$, $300 \mathrm{~s}$ and $300 \mathrm{~s}$, respectively.

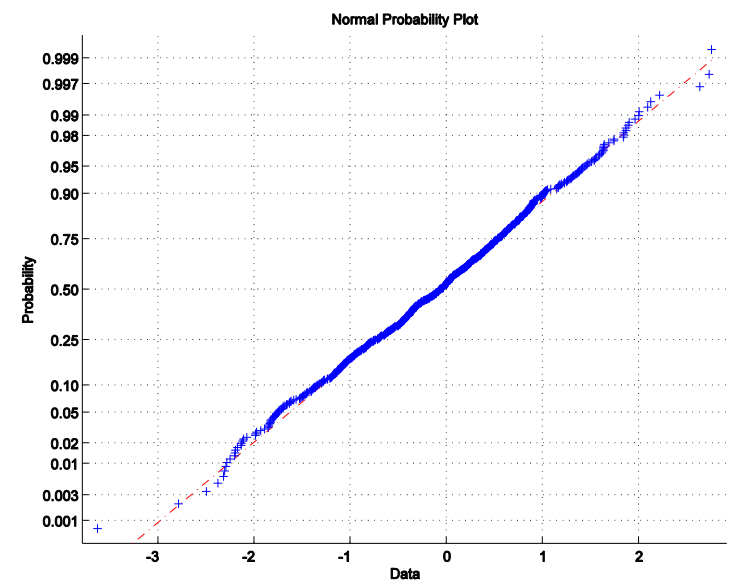

Figure 1. Normal Probability plot of w-test statistic for $p_{1}$ observations of GLONASS

\section{Evaluation of the Method Performance in Detetction and Identification of Outliers in Code Observations}

To evaluate the performance of the proposed algorithm, the following approach was carried out. First, several artificial errors were inserted at known epochs in the test data. Next, the proposed single-receiver single-satellite validation approach was performed. A check was carried out to examine whether the algorithm was able to detect the presence of the inserted errors at their known epochs. The inserted errors in code observations ranged between $0.6 \mathrm{~m}$ and $5 \mathrm{~m}$. These ranges were selected such that the minimum values equal the MDB, which is the minimum error that can be detected for each observation type with the chosen probabilities of false alaram and miss-detection, taken as 0.001 and 0.2 respectively. The MDBs are computed from the covariance matrix of the observations (see Teunissen 1998). The artificial errors were created using the MATLAB function "rand" such that they have random values that were bounded between $0.6 \mathrm{~m}$ and $5 \mathrm{~m}$. The inserted errors had almost a standard uniform distribution. Extra errors were added close to the lower bound to further test the method at this critical value. An example of the distribution of the inserted errors in p1 code errors for GLONASS satellites on 15/3/2012 is given in Figure 2.

In total, 5599 artificial errors were inserted in the code data. Table 1 gives the number of errors inserted in each system for each of the three test days followed by the percentage of successful detection of the inserted errors referenced to their known information. The errors were inserted for all observed 32 GPS satellites, 24 GLONASS satellites, and 4 Galileo satellites throughout the 24 hours of data for each of the test days and for all available frequencies. In general, the number of inserted errors were proportional to the number of

El-Mowafy, A. 2014. GNSS multi-frequency receiver single-satellite measurement validation method. GPS 
satellites observed in each system. The specific epochs and observations where these errors were inserted as well as their values were recorded. The results of Tables 1 are a function of the test significance level $(\alpha)$. Using a significance level for w-test statistics equals 0.001 , the significance level for the local over-all-model in the detetction test was computed using Baarda's B method (Baarda 1968), which assumes same probability for type II error (failure to reject a false null hypothesis) in both the detection and identification tests. In this study, this probability is taken equals 0.2 . The corresponding $\alpha$ for dual frequency observations was computed as 0.0052 , which is the case for GLONASS satellites and most GPS satellites. For triple-frequency observations, i.e. for Galileo satellites and GPS PRN 1 and 25, $\alpha$ was computed as 0.0123 .

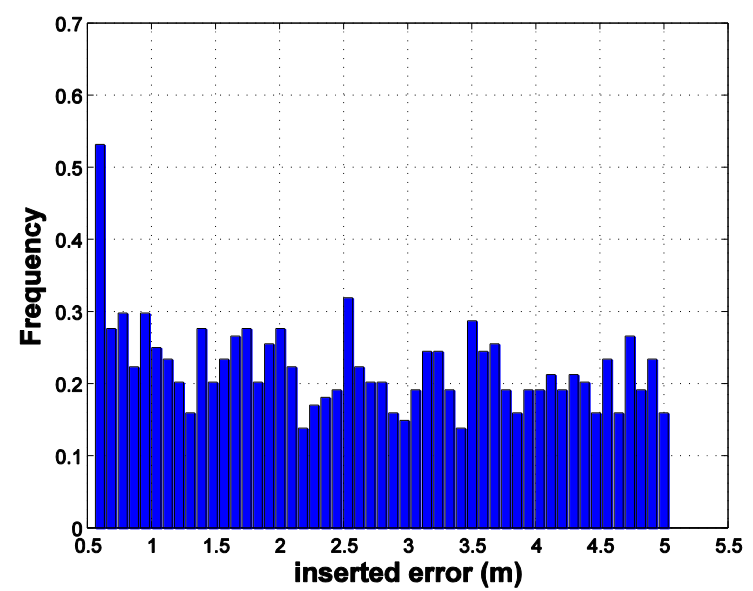

Fig. 2 Distribution of inserted errors for GLONASS p1 code measurements

Table 1 Percentage of epochs with detected code observation outliers (for all frequencies)

\begin{tabular}{|c|c|c|c|c|c|c|c|c|c|c|c|c|}
\hline System & \multicolumn{4}{|c|}{ GPS } & \multicolumn{4}{|c|}{ GLONASS } & \multicolumn{4}{|c|}{ Galileo } \\
\hline (m) & \#err. & $0.6-2$ & $2-3.5$ & $3.5-5$ & \#err. & $0.6-2$ & $2-3.5$ & $3.5-5$ & \#err. & $0.6-2$ & $2-3.5$ & $3.5-5$ \\
\hline $15 / 3 / 12$ & 922 & 75.6 & 92.5 & 98.7 & 795 & 72.3 & 83.9 & 92.5 & 148 & 94.3 & 98.8 & 98.9 \\
\hline $16 / 3 / 12$ & 895 & 76.3 & 93.1 & 95.4 & 798 & 76.0 & 86.2 & 93.5 & 158 & 91.7 & 95.2 & 100.0 \\
\hline $17 / 3 / 12$ & 920 & 80.2 & 92.3 & 95.7 & 810 & 78.0 & 90.5 & 95.2 & 153 & 97.0 & 100.0 & 100.0 \\
\hline
\end{tabular}

The success of detection summarized in Table 1 was separately given for errors within three bands ( $0.6 \mathrm{~m}$ to $2 \mathrm{~m}, 2 \mathrm{~m}$ to $3.5 \mathrm{~m}$, and the last $3.5 \mathrm{~m}$ to $5 \mathrm{~m}$ ). Detection results showed that the algorithm success rate increases as the error size increases and it was best for Galileo, followed by GPS and last GLONASS. For GPS, over the three test days, the successful detection of code outliers were on average $77.4 \%, 92.63 \%$, and $96.6 \%$ for the error ranges $0.6 \mathrm{~m}-2 \mathrm{~m}, 2 \mathrm{~m}-3.5$ $\mathrm{m}$, and $3.5 \mathrm{~m}-5 \mathrm{~m}$, respectively. The detection of code outliers for GLONASS were less than those of GPS. These percentages were significantly better with Galileo measurements, which on average were $94.3 \%, 98.0 \%$ and $99.63 \%$. This can be attributed to the better signal quality of GPS compared with GLONASS, and the enhanced quality of Galileo measurements as well as the more number of observations it has, which helps in strengthen application of the model.

For the epochs where detection was successful, a check was performed to examine whether the observations of the artificial errors can be correctly identified. Table 2 shows the overall percentage for identification of errors for the the same data and testing period that was discussed in the analysis of results of error detection. Successful error identification was assessed for each of the three error bands $0.6 \mathrm{~m}-2 \mathrm{~m}, 2 \mathrm{~m}-3.5 \mathrm{~m}$, and $3.5 \mathrm{~m}-5 \mathrm{~m}$. Results 
showed that the success rates of identifying outliers for the three error bands (for the epochs where errors were detected) were close and increased as error size increased. For GPS and Galileo the method was successful in identifying code outliers ranged from approximately $90 \%$ up to $99.5 \%$. The identifiication of outliers were almost $4 \%$ to $10 \%$ less for GLONASS. The variability of the success rate can be attributed to different observation precisions.

Table 2 Percentage of identification of code outliers (for all frequencies)

\begin{tabular}{c|ccc|ccc|ccc}
\hline System & \multicolumn{3}{|c|}{ GPS } & \multicolumn{3}{c|}{ GLONASS } & \multicolumn{3}{c}{ Galileo } \\
\hline$(\mathrm{m})$ & $0.6-2$ & $2-3.5$ & $3.5-5$ & $0.6-2$ & $2-3.5$ & $3.5-5$ & $0.6-2$ & $2-3.5$ & $3.5-5$ \\
\hline $15 / 3 / 12$ & 88.8 & 93.2 & 96.4 & 83.2 & 84.3 & 89.6 & 98.7 & 99.1 & 99.5 \\
\hline $16 / 3 / 12$ & 89.5 & 93.8 & 97.3 & 85.1 & 86.1 & 88.4 & 94.0 & 95.1 & 99.2 \\
\hline $17 / 3 / 12$ & 90.0 & 93.4 & 97.0 & 86.5 & 87.0 & 90.8 & 97.8 & 98.8 & 99.5 \\
\hline
\end{tabular}

The advantage of the method is demonstrated in another test by applying it in a single point positioning (SPP) mode using measurements only from the current Galileo constellation, which includes four IOV satellites, PRN 11, 12, 19 and 20. The used data spaned approximately three hours on 2/7/2013 (between 1:30 and 4:26 where the four satellites were simultaneously visible), collected at a CORS in Curtin University in a static mode with a sampling interval of $30 \mathrm{sec}$ using a Trimble Net R9 receiver. The data were screened epoch-by-epoch for detection and identification of code outliers. Such capability is not possible by current statistical validation methods, as they require redundancy of satellite measurements, which was not available in this test as only four satellites were used. However, with the single-receiver single-satellite method, data validation was possible owing to the fact that each satellite data were screened independently. A similar possible scenario can be experienced when working with other constellations in what is called "urban canyon". During the SPP processing of Galileo measurements only one code outlier was detected and removed. The coordinate differences along the East, North and Up directions between the epoch-by-epoch SPP least squares solution and the known station coordinates were computed and found to be bounded within $\pm 5 \mathrm{~m}$, indicating that no outliers were left in the data. Figures 3 and 4 show two examples of the time series of the ionosphere-free combination $m_{p_{i}}=p_{i}-\phi_{i}+2 \lambda_{i}^{2} \frac{\phi_{j}-\phi_{i}}{\lambda_{j}^{2}-\lambda_{i}^{2}}$ (which mainly gives multipath and code noise) for the Galileo satellites 11 and 12, where $i$ refers to the frequency E1 and $j$ referes to E5a frequency. As the figures show, no undetected outliers can be seen.

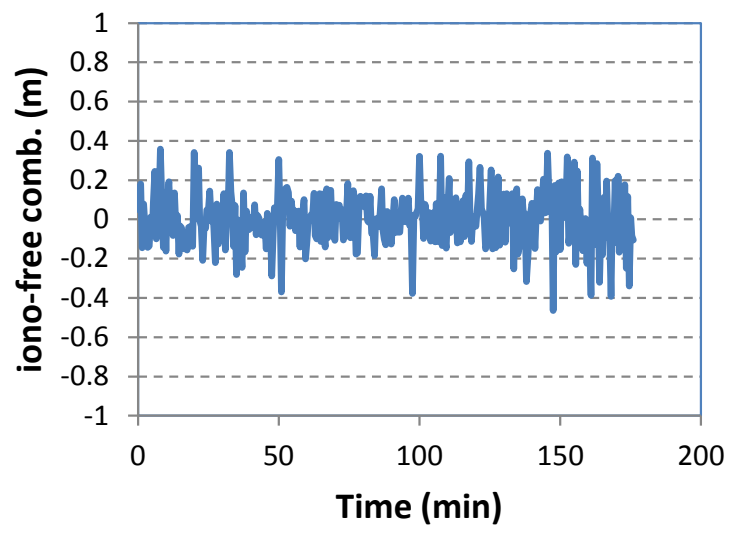

Fig. 3 iono-free combination for PRN 11

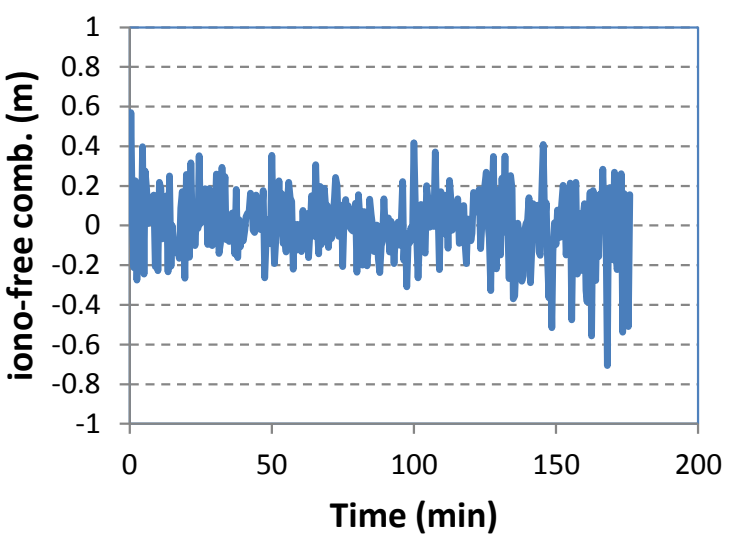

Fig. 4 iono-free combination for PRN 12 


\section{Evaluation of the Method Performance in Detection of Cycle Slips}

To evaluate the detection of cycle slips, a similar approach was carried out where 970 artificial cycle slips were inserted in the phase data of the three systems GPS, GLONASS and Galileo. The cycle slips were insterted for all available frequencies throughout the 24 hours of data for the three test days as given in Table 3. Global testing was performed to examine whether the algorithm was able to detect the presence of the inserted cycle slips. The inserted slips were random but ranged from one cycle to 6 cycles with almost a standard uniform distribution. Table 3 summarizes the numbers of inserted cycl slips and the percentage of their successful detection within two bands (1-3 cycles and 4-6 cycles). The table shows consistency in detection of cycle slips and that successful detection for GPS was on average approximately 95\% and 98\% respevctively for the two test bands. For Galileo, the latter had improved to $100 \%$. For GLONASS, the success rates were slightly less than GPS and Galileo for the reasons discussed earlier.

Table 3 Percentage of epochs with detected phase observation cycle slips (for all frequencies)

\begin{tabular}{c|ccc|ccc|ccc}
\hline System & \multicolumn{3}{|c|}{ GPS } & \multicolumn{3}{c|}{ GLONASS } & \multicolumn{3}{c}{ Galileo } \\
\hline cycles & \#slips & $1-3$ & $4-6$ & \#slips & $1-3$ & $4-6$ & \#slips & $1-3$ & $4-6$ \\
\hline $15 / 3 / 12$ & 144 & 95.8 & 98.6 & 142 & 92.9 & 95.8 & 40 & 95.0 & 100 \\
\hline $16 / 3 / 12$ & 142 & 94.4 & 98.6 & 138 & 91.3 & 95.7 & 39 & 94.7 & 100 \\
\hline $17 / 3 / 12$ & 144 & 94.4 & 97.2 & 141 & 91.5 & 95.8 & 40 & 95.0 & 100 \\
\hline
\end{tabular}

Another test for evaluation of the performance of the method in cycle slips detetction was executed by processing GPS data of 15/3/12 which contain the above slips using Bernese GNSS software version 5 in a percise point positioning (PPP) mode (see Dach et al. 2007 for the methods used in Bernese for detection of cycle slips). The number of cycle slips reported by Bernese software were compared with the inserted slips and with the slips detected by the single-receiver single-satellite method. Out of the 144 cycle slips in the data, Bernse software flaged 141 slips. Comparing this with the results of the proposed method, which had detected 140 cycle slips, shows that the perofrmance of the single-receiver single-satellite validation method was comparable to that of Bernse software for the test at hand.

\section{Summary}

A method that can be applied for real-time or post-mission quality control of GNSS measurements is presented using a single-receiver single-satellite DIA approach. The advantages of this approach include: it is applicable to any GNSS with any arbitrary number of frequencies, no need for the navigation message, the approach is able to detect faulty measurements for systems with a limited number of operational satellites, and there is no need for the determination of inter-system biases when using data from different constellations.

The capability of the proposed algorithm was evaluated for detection and identification of outliers in code observations and detection of cycle slips in phase observations of GPS, GLONASS and Galileo. Artificial errors were inserted in a data set that spans 3 days for all frequencies. The method was successful in detecting from $77.4 \%$ to $96.6 \%$ on average for the errors ranging between $0.6 \mathrm{~m}$ to $5 \mathrm{~m}$ in GPS observations. For GLONASS, the overall performance was slightly less than GPS, whereas for Galileo measurements, the average rates of successful detection ranged between $94.3 \%$ and $99.63 \%$. This can be attributed to differences in signal quality and number of observations, which helps in strengthen application of the model. Evaluation of the method performance in correct identification of code outliers

El-Mowafy, A. 2014. GNSS multi-frequency receiver single-satellite measurement validation method. GPS

Solutions. 18 (4): pp. 553-561.

Page 11 
showed that the method was successful in identifying $89.4 \%$ to $96.9 \%$ of GPS code outliers and with slightly lower performance for for GLONASS. For Galileo, the rates of successful identification of outliers were much better with average values between $96.8 \%$ and $99.4 \%$. Successful detection of artificil cycle slips in phase data was between $95 \%$ and $99 \%$ for GPS and Galileo and was a little less for GLONASS.

The advantage of the method is demonstrated in another test by using it in a single point positioning where measurements from only four IOV Galileo satellites were processed. Unlike other statistical testing methods, satellite redundancy was not needed as data of each satellite were screened independently. In another test, comparison between the number of cycle slip detected using the proposed method with that of Bernese software during PPP processing shows that they have a comparable performance.

Acknowledgments The author would like to thank Prof. P.J.G. Teunissen for proposing to carry out this study and for his suggestions and comments. The IRG fund received from Curtin University of Technology to carry out this research, project number 47606, is acknowledged.

\section{References}

Baarda W A (1968) Testing procedure for use in geodetic networks. Netherlands Geodetic Commission, Publications on Geodesy, New Series 2(5)

Blanch J, Walter T, Enge P (2010) RAIM with Optimal Integrity and Continuity Allocations under Multiple Failures. IEEE Transactions on Aerospace and Electronic Systems 46(3): 1235-1247

Blewitt G (1990) An Automatic Editing Algorithm for GPS Data. Geophysical Research Letters 17(3): 199-202

Banville S, Langley R (2010) Instantaneous Cycle-Slip Correction for PPP. Navigation 57(4): 325-334

Dach R, Hugentobler U, Fridez P, Meindl M (2007) Bernese GPS Software Version 5.0. Publications of Astronomical Institute, University of Bern.

De Bakker PF, Van der Marel H, Teunissen P JG (2009a) The Minimal Detectable Bias for GNSS Observations with a Single Receiver Setup and a Geometry-Free Model. Proc. ENC-GNSS 2009, Naples, Italy, 3-6 May 2009

De Bakker PF, Van der Marel H, Tiberius CCJM (2009b) Geometry-free undifferenced, single and double differenced analysis of single frequency GPS, EGNOS and GIOVE-A/B measurements. GPS Solutions 13: 305-314

De Jong K, Teunissen PJG (2000) Minimal Detectable Biases of GPS Observations for a Weighted Ionosphere. Earth, Planets and Space 52: 857-862

De Jong K., Van der Marel H, Jonkman N (2001) Real-Time GPS and Glonass Integrity Monitoring and Reference Station Software. Physics and Chemistry of the Earth (A) 26(6-8): 545-549

El-Mowafy A, Teunissen PJG, Odijk D (2010) Single-Receiver Single-Channel Real-Time Validation of GPS, GLONASS, Galileo and COMPASS Data. Proc. International Symposium on GPS/GNSS, Taipei, Taiwan, 26-28 Oct. 2010

El-Mowafy A (2009) An Alternative Post-Processing Relative Positioning Approach Based on Precise Point Positioning. Journal of Surveying Engineering 135(2): 56-65

Ene A, Blanch J, Powell JD (2007) Fault Detection and Elimination for Galileo-GPS Vertical Guidance, Proc. of the Institute of Navigation National Technical Meeting, San Diego, CA, 22-24 January 2007

Euler, H-J, and C.C Goad (1991) On optimal Filtering of GPS Dual-Frequency Observations without Using Orbit Information. Bulletin Géodésique 65: 130-143

Farrell, J. L. and Van Graas. F. (1992). Statistical validation for GPS integrity test, NAVIGATION, 39(2): 205-216.

Gelb A (1974) Applied Optimal Estimation. Massachusetts Institute of Technology Press, Cambridge, Massachusetts

El-Mowafy, A. 2014. GNSS multi-frequency receiver single-satellite measurement validation method. GPS

Solutions. 18 (4): pp. 553-561.

Page 12 
Guo J, Lu M, Cui X, Feng Z (2011) A New RAIM Algorithm for Triple-Frequency GNSS Receivers, Proc. the 2011 International Technical Meeting of The Institute of Navigation San Diego, CA, 24 - 26 January 2011: 271 - 278

Kaplan ED (2006) Understanding GPS - Principles and Applications. 2nd ed. Boston: Artech House

Kim D, Langley RB (2002) Instantaneous Real-Time Cycle-Slip Correction for Quality Control of GPS Carrier-phase Measurements. Navigation 49(4): 205-222

Kok J (1984) On Data Snooping and Multiple Outlier Testing, Vol. 30 of NOAA technical report NOS.: NGS, U.S. Department of Commerce, National Oceanic and Atmospheric Administration, National Ocean Service, Charting and Geodetic Services, 61p.

Lee Y (2012) New Advanced RAIM with Improved Availability for Detecting Constellation-wide Faults Using Two Independent Constellations, Navigation 60(1): 71-83.

Leick A (2004) GPS satellite surveying, 3rd ed. Wiley, New York

Neri P, Azoulai L, Macabiau C (2011) Study of the Temporal Behavior of GPS/GALILEO NSE and RAIM for LPV200. Proc. ION GNSS 2011, Oregon, Portland, 19-23 September 2011

Teunissen PJG, de Bakker PF (2012) Next Generation GNSS Single Receiver Cycle Slip Reliability, Proc. VII Hotine-Marussi Symposium on Mathematical Geodesy, International Association of Geodesy Symposia 137: 159-164

Teunissen PJG (2006) Testing theory; an introduction. 2nd ed., Delft VSSD, The Netherlands

Teunissen PJG (1998) Minimal detectable biases of GPS data. Journal of Geodesy 72: 236-244

Teunissen P.J.G., and Kleusberg A. (1998). GPS for Geodesy, 2nd ed., Springer, NY.

Teunissen PJG (1990) Quality Control in Integrated Navigation Systems. IEEE Aerospace and Electronic Systems Magazine 5(7): 35-41

\section{Author Biography}

Ahmed El-Mowafy is a Senior Lecturer, Department of Spatial Sciences, Curtin University, Australia. He has obtained his Ph.D. from the University of Calgary, Canada, in 1995. He has extensive publications in precise positioning and navigation using GNSS, quality control and integrity monitoring, estimation theory, and attitude determination. He is a member of the Editorial Advisory Board of the Journal of Navigation, and a member of the Editorial Board of the International Journal of Geoscience Research. 